\title{
Deterministic Equations of Motion and Dynamic Critical Phenomena
}

\author{
B. Zheng*†, M. Schulz* and S. Trimper* \\ * Universität - Halle, 06099 Halle, Germany \\ $\dagger$ Universität - GH Siegen, 57068 Siegen, Germany
}

\begin{abstract}
Taking the two-dimensional $\phi^{4}$ theory as an example, we numerically solve the deterministic equations of motion with random initial states. Short-time behavior of the solutions is systematically investigated. Assuming that the solutions generate a microcanonical ensemble of the system, we demonstrate that the second order phase transition point can be determined already from the short-time dynamic behavior. Initial increase of the magnetization and critical slowing down are observed. The dynamic critical exponent $z$, the new exponent $\theta$ and the static exponents $\beta$ and $\nu$ are estimated. Interestingly, the deterministic dynamics with random initial states is in a same dynamic universality class of Monte Carlo dynamics.
\end{abstract}

PACS: 05.20.-y, 02.60.Cb, 64.60.Ht, 11.10.-z 
It is believed that statistical mechanics is originated from the fundamental equations of motion for many body systems or field theories, even though up to now there exists not a general proof. For classical systems, equations of motion are deterministic. Statistical ensembles are expected to be effective description of the systems. For quantum systems the situation is similar. In any case, practically it remains open whether solutions of the fundamental equations of motion really yield the same results as statistical mechanics, e.g., see Refs. [15].

With recent development of computers, to solve numerically equations of motion gradually becomes possible. This has attracted much attention of scientists in different areas. For example, recently the $O(N)$ vector model and $X Y$ model have been numerically solved [6 8]. Noting that energy is conserved during time evolution, solutions of equations of motion actually generate a microcanonical ensemble. Making the time average of the observables, introducing standard techniques developed in statistical mechanics for the canonical ensemble, phase transition points and critical exponents have been estimated. The results are in agreement with those obtained from a canonical ensemble in statistical mechanics.

In principle, the deterministic equations of motion should describe not only equilibrium properties but also dynamic properties of the systems. In statistical mechanics, dynamics is approximately given by some effective stochastic equations of motion, typically at mesoscopic level, e.g. Langevin equations or Monte Carlo algorithms. For stochastic dynamics, critical slowing down and dynamic scaling around the critical point are characteristic properties. It has long been challenging whether stochastic dynamics describes correctly the real physical world. It is important and interesting to study dynamic properties of the fundamental deterministic equations of motion and whether deterministic dynamics and stochastic dynamics are in a same universality class.

On the other hand, in recent years much progress has been achieved in stochastic dynamics. For a long time it is known that around the critical point there exists universal scaling behavior in the long-time regime of the dynamic evolution. This is more or less due to the divergent correlation time induced by the divergent spatial correlation length. Short-time behavior is believed to depend on microscopic detail. However, in recent years it is discovered that universal scaling behavior emerges already in the macroscopic short-time regime, after a time scale $t_{m i c}$ which is sufficiently large in the microscopic sense [9 15] (for a review, see Ref. [16]). Important is that one should take care of the macroscopic initial conditions carefully. For example, dynamic magnetic systems with an initial state of very high temperature and small magnetization, the short-time dynamic scaling for the $k$ th moment of the magnetization is written as [9]

$$
M^{(k)}\left(t, \tau, L, m_{0}\right)=b^{-k \beta / \nu} M^{(k)}\left(b^{-z} t, b^{1 / \nu} \tau, b^{-1} L, b^{x_{0}} m_{0}\right) .
$$

Here $\tau=\left(T-T_{c}\right) / T_{c}$ is the reduced temperature, $\beta, \nu$ are the well known static critical exponents and $z$ is the dynamic exponent, while the new independent exponent $x_{0}$ is the scaling dimension of the initial magnetization $m_{0}$. The parameter $b$ is an arbitrary rescaling factor and $L$ is the lattice size. For the dynamics of model A, a prominent property of the short-time dynamic scaling is that the magnetization undergoes a critical initial increase at early time [9,13,17, 18,

$$
M(t) \sim m_{0} t^{\theta}
$$


where the exponent $\theta$ is related to $x_{0}$ by $\theta=\left(x_{0}-\beta / \nu\right) / z$.

More interesting and important is that the static exponents $\beta, \nu$ and the dynamic exponent $z$ in the short-time scaling form (四) take the same values as they are defined in equilibrium or the long-time regime. This fact makes it possible to determine all these exponents already in the short-time regime of the dynamic evolution [16]. Since now the measurements are carried out at early time, the method is free of critical slowing down. The short-time dynamic scaling is not only conceptually interesting but also practically important. To clarify whether the short-time dynamic scaling is a fundamental phenomenon of the real physical world becomes urgent.

The purpose of this letter is to study whether there exists short-time universal scaling behavior in the dynamics described by the deterministic equations of motion and meanwhile determine the critical point and all the static and dynamic exponents.

The model we choose is the two-dimensional $\phi^{4}$ theory. The reason is that the statics of this model is known in a same universality class of the two-dimensional Ising model and there exist already some numerical results [6]. The Hamiltonian of the model on a square lattice is

$$
H=\sum_{i}\left[\frac{1}{2} \pi_{i}^{2}+\frac{1}{2} \sum_{\mu}\left(\phi_{i+\mu}-\phi_{i}\right)^{2}-\frac{1}{2} m^{2} \phi_{i}^{2}+\frac{1}{4 !} \lambda \phi_{i}^{4}\right]
$$

with $\pi_{i}=\dot{\phi}_{i}$ and it leads to the equations of motion

$$
\ddot{\phi}_{i}=\sum_{\mu}\left(\phi_{i+\mu}+\phi_{i-\mu}-2 \phi_{i}\right)+m^{2} \phi_{i}-\frac{1}{3 !} \lambda \phi_{i}^{3} .
$$

In the dynamic evolution governed by equations (雨), energy is conserved. The solutions are assumed to generate a microcanonical ensemble. The temperature could not be introduced externally as in a canonical ensemble, but could only be defined internally as the averaged kinetic energy. In our short-time dynamic approach, the total energy is actually a even more convenient controlling parameter of the system, since it is conserved and can be input from the initial state. Therefore, from now $\tau$ in the scaling form (1) will be understood as a reduced energy density $\left(\epsilon-\epsilon_{c}\right) / \epsilon_{c}$. Here $\epsilon_{c}$ is the critical energy density corresponding to a second order phase transition.

The order parameter of the $\phi^{4}$ theory is the magnetization. The time-dependent magnetization $M \equiv M^{(1)}(t)$ and its second moment $M^{(2)}$ are defined as

$$
M^{(k)}(t)=\frac{1}{L^{2 k}}\left\langle\left[\sum_{i} \phi_{i}(t)\right]^{(k)}\right\rangle, \quad k=1,2 .
$$

The average for observables is only over initial configurations. In the short-time regime of the dynamic evolution, spatial correlation length is small, one can easily realize that $M^{(2)} \sim 1 / L^{d}$. From the scaling form (1), we obtain at the critical point for $m_{0}=0$ 10,18, 16.

$$
M^{(2)}(t) \sim t^{(d-2 \beta / \nu) / z} .
$$

Another interesting observable is the auto-correlation function 


$$
A(t)=\frac{1}{L^{2}} \sum_{i}<\phi_{i}(0) \phi_{i}(t)>.
$$

From the calculations based on Langevin equations, at the critical point the auto-correlation $A(t)$ with $m_{0}=0$ presents a power law behavior [19]

$$
A(t) \sim t^{-d / z+\theta}
$$

The power law behavior indicates critical slowing down. The interesting point is that the new exponent $\theta$ describing the initial increase of the magnetization also appears here.

Our strategy is that from initial increase of the magnetization, Eq. (2), we measure the exponent $\theta$. Taking $\theta$ as an input, from the power law decay of the auto-correlation, Eq. (8), we extract the dynamic exponent $z$. Then from power law behavior of the second moment, Eq. (6), we obtain the static exponent $\beta / \nu$. Finally, the critical point and the remaining exponent $\nu$ are determined as follows. Assuming $L$ is sufficiently large and $t^{x_{0} / z}$ is small enough, one can deduce from scaling form (1)

$$
M(t)=m_{0} t^{\theta} F\left(t^{1 / \nu z} \tau\right) .
$$

When $\tau=0$, Eq. (2) is recovered. When $\tau \neq 0$, the power law behavior will be modified. Therefore, searching for a energy density which gives the best power law behavior for the magnetization, one determines the critical point [16]. The exponent $1 / \nu z$ can be extracted from the logarithmic derivative [16]

$$
\left.\partial_{\tau} \ln M\left(t, \tau, m_{0}\right)\right|_{\tau=0} \sim t^{1 / \nu z} .
$$

For stochastic dynamics in a canonical ensemble, to obtain the scaling form (1) and (8) the initial state is taken to be a very high temperature state. Therefore, for deterministic dynamics we consider a random initial state with zero or small magnetization. For simplicity, we also set initial kinetic energy to be zero, i.e. $\dot{\phi}_{i}(0)=0$. Practically we proceed as follows. We first fix the magnitude of the initial field to be a constant $c,\left|\phi_{i}(0)\right|=c$, then randomly give the sign to $\phi_{i}(0)$ with the restriction of a fixed magnetization in unit of $c$, and finally the constant $c$ is determined by the given energy. Following Ref. [6], we take parameters $m^{2}=2$. and $\lambda=0.6$.

To solve these equations of motion numerically, we simply discretize $\ddot{\phi}_{i}$ by $\left(\phi_{i}(t+\Delta t)+\right.$ $\left.\phi_{i}(t-\Delta t)-2 \phi_{i}(t)\right) /(\Delta t)^{2}$. After an initial configuration is prepared, we update the equations of motion until $t=500$. Then we repeat the procedure with another initial configuration. In our calculations, we use a fairly large lattice $L=256$ and samples of initial configurations for average range from 4500 to 10000 depending on initial magnetization $m_{0}$. Errors are simply estimated by dividing total samples into two or three groups. Extra calculations with $L=128$ show that the finite size effect for $L=256$ is already negligible small.

Compared with numerical solutions in the long-time regime, the finite $\Delta t$ effect in the short-time dynamic approach is less severe, since our updating time is limited. Our results are presented with $\Delta t=0.05$. We have also performed some simulation with $\Delta t=0.01$ and confirmed that the finite $\Delta t$ effect has been rather small. In any case, however, since we have this small $\Delta t$, the calculations are much more lengthy than standard Monte Carlo simulations. Therefore, the purpose of this paper is to explore new physics rather than to pursue high accuracy of measurements. 
In order to determine the critical point, we perform simulations with non-zero initial magnetization $m_{0}$ for a couple of energy densities in the critical regime. In Fig. 1, time evolution of the magnetization with $m_{0}=0.015$ for parameters $m^{2}=2$. and $\lambda=0.6$ has been plotted with solid lines for three energy densities $\epsilon=20.7,21.1$ and 21.5 in $\log$ - $\log$ scale. To show the universal behavior clearly, we have cut the data for $t<t_{\text {mic }} \approx 50$. Even looking by eyes, one could realize that the magnetization of $\epsilon=21.1$ has rather good power law behavior. Actually, careful analysis of the data between $t=50$ and 500 leads to the critical energy density $\epsilon_{c}=21.11(3)$. This agrees well with $\epsilon_{c}=21.1$ given by the Binder cumulant in equilibrium in Ref. [6].

At $\epsilon_{c}$, one measures the exponent $\theta=0.146(3)$. Accurately speaking, however, the exponent $\theta$ is defined in the limit $m_{0} \rightarrow 0$. In general, for finite $m_{0}$ the exponent $\theta$ may show some $m_{0}$-dependence [16]. Therefore, we have performed another simulation with $m_{0}=0.009$ at $\epsilon_{c}$. The magnetization is also displayed in Fig. 1 with a dashed line. The corresponding exponent is $\theta=0.158(2)$. If we linearly extrapolate the results to $m_{0}=0$, we obtain the final value $\theta=0.176(7)$.

With the critical energy in hand, we set $m_{0}=0$ and proceed to measure the autocorrelation $A(t)$ and second moment $M^{(2)}(t)$. In Fig. 2, $A(t)$ and $M^{(2)}(t)$ are displayed in log-log scale with a solid and dashed line respectively. In order to show some typical microscopic behavior within $t_{m i c}$, here we present the data from a relatively early time $t=10$. From the figure we see clearly that at very early time, the curves do not have power law behavior, typically oscillating a bit. However, after $t>t_{\text {mic }} \approx 50$, both curves nicely stabilize to power law behavior. From the data for $t>50$, we measure the exponents $d / z-\theta=0.755(5)$ and $(d-2 \beta / \nu) / z=0.819(12)$.

Finally, to complete the estimate of the exponents, we calculate approximately the derivative of the magnetization with respect to the energy density with the data for Fig. 1. In Fig. 3, the logarithmic derivative of the magnetization is plotted in log-log scale at the critical point. As usual, the fluctuation here is larger [16]. The power law behavior is also not as clean as that of the previous observables. The slope of the curve tends to be smaller as the time $t$ evolves. To improve this situation, probably we need to perform simulations with energy densities closer to $\epsilon_{c}$ and with high statistics and/or to consider corrections to scaling. This requests very much computer times. In any case, from the slope between $t=200$ and 500 , we estimate $1 / \nu z=0.492(26)$.

In Table $\mathbb{1}$, we summarize all the values of the critical exponents discussed above. From these values, we can estimate the critical exponents $z, 2 \beta / \nu$ and $\nu$. The results are also listed in Table \&. For comparison, the exact values of $2 \beta / \nu$ and $\nu$ for the Ising model and available results for other exponents measured from a similar dynamic process with standard Monte Carlo dynamics are also given in Table 1 . Remarkably, not only the static exponents, but also the dynamic exponents of the $\phi^{4}$ theory are very close to those of the Ising model with standard Monte Carlo dynamics. The exponent $\theta$ shows some percents of difference. However, taking into account that the measurements of $\theta$ are not so easy, especially the extrapolation to $m_{0}=0$ limit may induce some systematic errors, we would consider that the exponent $\theta$ for both model are the same. Therefore, we conclude that the $\phi^{4}$ theory described by the deterministic equations of motion are in a same static as well as dynamic universality class of the Ising model with standard Monte Carlo dynamics.

Why is the deterministic dynamics in a same universality class of the stochastic dynam- 
ics? The origin may be traced back to the random initial state and the kinetic energy term in the Hamiltonian. The random initial configuration of $\left\{\phi_{i}\right\}$ induced stochastically evolving kinetic energy. The stochastic kinetic energy serves as a kind of noises or heat bath to the potential energy. This is similar to the stochastic dynamics with a canonical ensemble, where the Hamiltonian is simply the potential energy term here.

In conclusions, we have numerically solved the deterministic equations of motion for the two-dimensional $\phi^{4}$ theory. Short-time universal behavior is found. Based on the short-time dynamic scaling form, the critical point and all the static and dynamic critical exponents are determined. The values of the static exponents agree with those of the Ising model. More interestingly, both the dynamic exponents $z$ and $\theta$ also coincide with those of the kinetic Ising model induced by the standard Monte Carlo algorithms (heat-bath and Metropolis et al). Especially, the dynamic exponent $z=2.15(2)$ is very far from $z=1$, which is the expectation from the naive deterministic viewpoint. These results show that, on the one hand, the deterministic equations of motion indeed can describe statistical properties of the systems both in statics and dynamics, on the other hand, the Monte Carlo dynamics can be a good effective description of the real physical dynamics, at least in some cases.

Since the measurements are carried out in the short-time regime of the dynamic evolution, our dynamic approach does not suffer from critical slowing down. Furthermore, the errors induced by a finite $\Delta t$ in our measurements are also limited. It is challenging to derive analytically the short-time dynamic scaling from deterministic equations of motion. Extension of the present work to quantum systems would be very interesting.

Acknowledgements: Work supported in part by the Deutsche Forschungsgemeinschaft; Schu 95/9-1 and SFB 418. 


\section{REFERENCES}

[1] E. Fermi, J. Pasta and S. Ulam, in Collected Papers of Enrico Fermi, edited by E. Segré (Univ. Chicago, Chicago, 1965).

[2] J. Ford, Phys. Rep. 213, 271 (1992).

[3] D. Escande, H. Kantz, R. Livi and S. Ruffo, J. Statist. Phys. 76, 605 (1994).

[4] M. Antoni and S. Ruffo, Phys. Rev. E52, 2361 (1995).

[5] Y. Elskens and M. Antoni, Phys. Rev. E55, 6575 (1997).

[6] L. Caiani, L. Casetti and M. Pettini, J. Phys. A31, 3357 (1998).

[7] L. Caiani, L. Casetti, C. Clementi, G. Pettini, M. Pettini and R. Gatto, Phys. Rev. E57, 3886 (1998).

[8] X. Leoncini and A.D. Verga, Phys. Rev. E57, 6377 (1998).

[9] H. K. Janssen, B. Schaub and B. Schmittmann, Z. Phys. B 73, 539 (1989).

[10] D. A. Huse, Phys. Rev. B 40, 304 (1989).

[11] D. Stauffer, Physica A 186, 197 (1992).

[12] K. Oerding and H. K. Janssen, J. Phys. A26, 3369,5295 (1993).

[13] Z.B. Li, U. Ritschel and B. Zheng, J. Phys. A27, L837 (1994).

[14] S.N. Majumdar, A.J. Bray, S. Cornell and C. Sire, Phys. Rev. Lett. 77, 3704 (1996).

[15] H.J. Luo, L. Schülke and B. Zheng, Phys. Rev. Lett. 81, 180 (1998).

[16] B. Zheng, Int. J. Mod. Phys. B12, 1419 (1998), review article.

[17] P. Grassberger, Physica A 214, 547 (1995).

[18] L. Schülke and B. Zheng, Phys. Lett. A 204, 295 (1995).

[19] H. K. Janssen, in From Phase Transition to Chaos, edited by G. Györgyi, I. Kondor, L. Sasvári and T. Tél, Topics in Modern Statistical Physics (World Scientific, Singapore, 1992). 


\section{FIGURES}

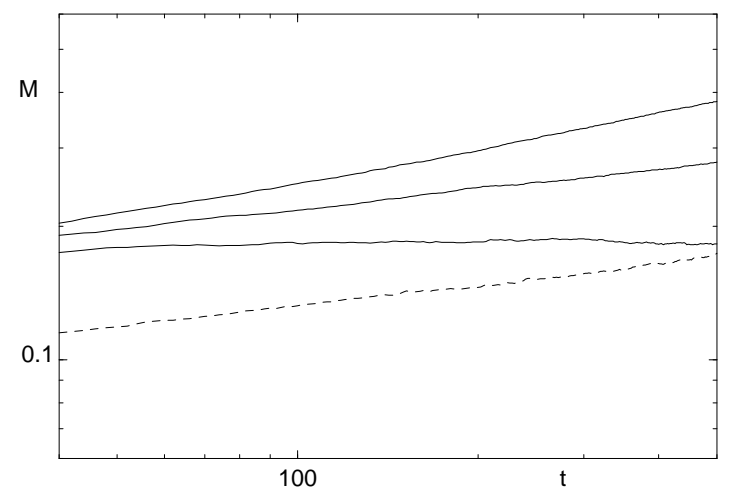

FIG. 1. Time evolution of the magnetization in log-log scale. Solid lines are for $m_{0}=0.015$ with energy densities $\epsilon=20.7,21.1$ and 21.5 (from above), while the dashed line is for $m_{0}=0.009$ with $\epsilon_{c}=21.11$.

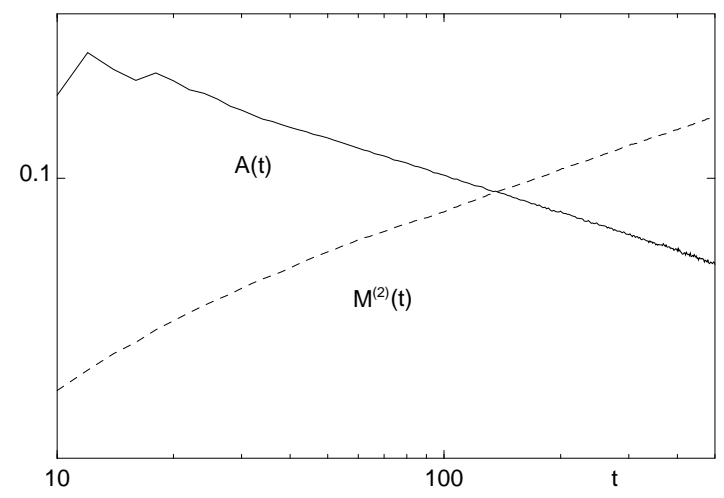

FIG. 2. The auto-correlation $A(t)$ (solid line) and second moment $M^{(2)}(t)$ (dashed line) with $m_{0}=0$ at the critical point in log-log scale.

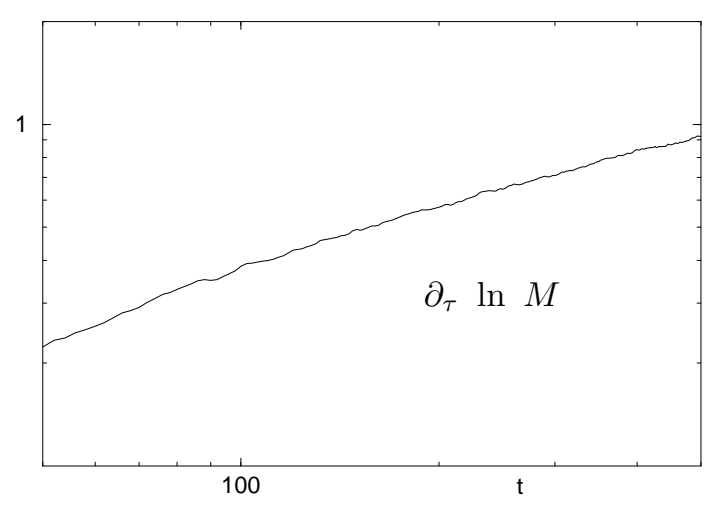

FIG. 3. Logarithmic derivative of the magnetization at the critical point in log-log scale. 


\section{TABLES}

\begin{tabular}{cccccccc}
\hline \hline & $\theta$ & $d / z-\theta$ & $(d-2 \beta / \nu) / z$ & $1 / \nu z$ & $z$ & $2 \beta / \nu$ & $\nu$ \\
$\phi^{4}$ & $0.176(7)$ & $0.755(5)$ & $0.819(12)$ & $0.492(26)$ & $2.148(20)$ & $0.24(3)$ & $0.95(5)$ \\
Ising & $0.191(1)$ & $0.737(1)$ & $0.817(7)$ & & $2.155(3)$ & $1 / 4$ & 1 \\
\hline \hline
\end{tabular}

TABLE I. The critical exponents measured for the $\phi^{4}$ theory in comparison with those of the Ising model. The values of $2 \beta / \nu$ and $\nu$ for the Ising model are exact, while others are taken from Table 2 in Ref. [16]. 